\title{
EFEITO DO CONSUMO DE ÁGUA RICA EM SAIS DE VANÁDIO SOBRE A GLICEMIA DE RATOS DIABÉTICOS
}

\author{
Effect of vanadium-rich water intake on glycemia of diabetic rats
}

\author{
Claudia Bianca Kimura ${ }^{1}$ \\ Clodoaldo Rogériodos Reis ${ }^{2}$ \\ Luiz Cláudio Fernandes ${ }^{3}$
}

\section{Resumo}

Diabetes mellitus é uma doença caracterizada principalmente por hiperglicemia crônica. Sua etiologia pode estar associada à produção insuficiente ou resistência à insulina. O desenvolvimento de terapias alternativas para a diabetes é muito importante. Vários compostos de vanádio vêm sendo indicados no tratamento para da diabetes, devido ao seu efeito mimético ao da insulina. Muitos pacientes afirmam melhora de seu estado diabético ao consumirem tal água. Este estudo investigou o efeito do consumo de água rica em vanádio, proveniente de Ibirá (SP), sobre a glicemia de ratos induzidos a diabetes com aloxana. A concentração de sais de vanádio na água foi de $0,042 \mathrm{mg} / \mathrm{L}$. Após ingestão da água por 16 dias pelos animais com diabetes tipo I, observou-se redução na perda de massa corporal e da polidipsia quando em comparação à dos animais sem tratamento, contudo a glicemia não se alterou significativamente.

Palavras-chave: Diabetes mellitus; Vanádio; Aloxana; Efeito mimético à insulina.

\section{Abstract}

Diabetes mellitus is a disease characterized mainly by chronic hyperglycemia where its ethiology it has been associated to insufficient production or resistance to insulin. Development of alternative therapies for diabetes treatment is quite important and some vanadium compounds have being used for diabetes treatment due to its insulin mimetic effect. As matter of fact many patients had stated improvement of their diabetes status further consumption of such water. This study investigated the effect of water intake from Ibirá (SP), rich in vanadium compounds in diabetic rats for 16 days. Vanadium concentration in the water was by $0.042 \mathrm{mg} / \mathrm{l}$. After consuming of such water for 16 days, diabetic rats presented reduction of water intake and also in the body weight loss when compared to no treated animals, however there was not significant alteration on glycemia.

Keywords: Diabetes mellitus; Vanadium; Alloxan; Insulin mimetic effect.

\footnotetext{
Graduação, Bacharelado em Biologia, Pontifícia Universidade Católica do Paraná, Curitiba, PR. e-mail: claudiabk@gmail.com

Ibirá, SP. e- mail: clodoaldorogerio@hotmail.com

Doutor, Departamento de Fisiologia, Setor de Ciências Biológicas, Universidade Federal do Paraná, 81530-990, Curitiba, PR. e-mail: lcfer@ufpr.br
} 
Introdução

A insulina é um hormônio produzido pelas células b-pancreáticas e importante reguladora na homeostasia da glicose. De forma coordenada, atua em eventos celulares que regulam os efeitos metabólicos e de crescimento $(1,2,3)$.

Deficiência absoluta ou relativa na secreção de insulina e/ou resistência dos tecidos alvos à ação da insulina podem resultar na doença diabetes mellitus. Diabetes mellitus leva a um estado metabólico anormal, caracterizado por hiperglicemia crônica e desenvolvimento de patologia microvascular específica na retina, glomérulo renal e nervos periféricos $(4,5,6)$.

A diabetes dependente de insulina ou diabetes tipo I é doença crônica resultante da destruição específica de células b-pancreáticas, por processo autoimune $(7,8,9)$. A diabetes tipo II é a forma mais freqüente, afetando entre $90 \%$ a $95 \%$ dos indivíduos diabéticos. Esta resulta da interação de vários fatores genéticos e ambientais, onde os indivíduos desenvolvem resistência progressiva à insulina $(7,10)$.

A insulina é utilizada para tratamento de todos os casos de diabetes tipo I e também em muitos pacientes com diabetes tipo II, para redução da hiperglicemia severa, devido à falha progressiva da célula $\mathrm{b}(1,9,11)$. A disponibilidade de substitutos para insulina tais como os fármacos sulfonilúreas, tiazolidinedionas, metaformina e inibidores de b-glicosidase (acarbose) e sais de vanádio podem ser de extrema importância no tratamento da diabetes mellitus. Vários compostos de vanádio têm sido indicados como agentes que possuem grande potencial para o tratamento, por melhorarem a hiperglicemia e a homeostasia anormal da glicose em modelos animais de diabetes tipo I e II (9).

O vanádio é elemento traço essencial pertencente ao grupo de elementos de transição. Existe em inúmeros estados diferentes de oxidação e possui tendência para formar íons complexos. Em geral, estes se situam entre três grandes categorias, incluindo complexos de peroxovanádio, compostos de vanádio orgânicos e as formas predominantes, os sais de vanádio inorgânicos (vanadato ou vanadil) $(12,13)$. Apesar de ser muito eficaz em controlar concentrações elevadas de glicose sangüínea, a toxicidade do vanádio pode limitar seu papel de agente terapêutico para o tratamento do diabetes mellitus $(11,14,15)$.
Nos arredores de Ibirá, SP, há fontes de águas naturais ricas em sais de vanádio, que ultrapassam as concentrações encontradas em outras regiões do país. Pacientes diabéticos têm consumido água desta região e afirmam ter melhora de seu estado diabético. Até a presente data, não há relatos de trabalhos científicos que, de fato, comprovem ou não esta propriedade "anti"-diabética de tal água. Este trabalho se propôs a investigar o efeito do consumo desta água rica em vanádio, em modelo animal de diabetes tipo I.

\section{Material e métodos}

\section{Animais}

Para o presente estudo, foram utilizados ratos Wistar (Ratus norvergicus norvergicus Berbenhout, 1769) machos, pesando aproximadamente $300 \mathrm{~g}$, obtidos no Biotério do Setor de Ciências Biológicas da Universidade Federal do Paraná. Estes foram mantidos em sala sob ciclo claro/escuro 12/12 horas, com água e ração monitorados diariamente. Os ratos foram acondicionados em caixas de plástico com cinco animais cada e divididos em grupo controle (C); grupo diabético (D); grupo diabético tratado com insulina $(\mathrm{D}+\mathrm{I})$; grupo diabético tratado com vanádio $(\mathrm{D}+\mathrm{V})$ e grupo diabético tratado com vanádio e insulina $(\mathrm{D}+$ $\mathrm{V}+\mathrm{I}$ ). A massa corporal dos ratos foi monitorada a cada sete dias em balança digital Urano.

Os procedimentos utilizados foram aprovados pelo Comitê de Ética da UFPR (certificado $\mathrm{n}^{\circ} 06$ ) e seguiram os princípios éticos estabelecidos pelo Código Brasileiro de Experimentação Animal (COBEA) e exigências estabelecidas no "Guide for the care and use of experimental animals" (Canadian Council on Animal Care).

\section{Indução da diabetes tipo I}

Os ratos foram sedados com éter etílico e intravenosamente foi administrada aloxana $(45 \mathrm{mg} /$ $\mathrm{kg}$ Sigma St. Louis, EUA) diluída em solução salina $0,9 \%$. Jejum de 12 horas precedeu as injeções que foram efetuadas em dois dias alternados, onde somente após uma hora a partir das injeções os animais voltaram a receber água e comida. Foram considerados diabéticos os animais que apresentaram concentração plasmática de glicose igual ou superior a $300 \mathrm{mg} / \mathrm{dL}$. 


\section{Determinação da glicemia}

Os animais foram sedados com éter etílico e aproximadamente $0,2 \mathrm{~mL}$ de sangue foi coletado da cauda. A determinação da concentração sérica de glicose circulante foi realizada pelo método enzimático colorimétrico, utilizando o kit glicose PAP Liquiform da Labtest Diagnóstica $(16,17)$, seguindo as instruções do fabricante.

\section{Água rica em vanádio}

A água foi coletada em algumas fontes em Ibirá, SP, na propriedade do senhor Clodoaldo Rogério do Reis, que gentilmente as cedeu para a referida pesquisa. A análise química da água foi realizada pelo Prof. Dr. José Marcus Godoy, do Departamento de Química da Pontifícia Universidade Católica do Rio de Janeiro. A concentração de vanádio encontrada na água foi $0,042 \mathrm{mg} / \mathrm{L}$, cujo laudo técnico afirmou ser água mineral alcalina bicarbonatada vanádica fluoretada e fracamente radioativa na fonte.

\section{Tratamento da diabetes}

O tratamento teve início após a confirmação do estado diabético por meio da dosagem glicêmica e se estendeu por um período de 16 dias. Nos ratos dos grupos $\mathrm{D}+\mathrm{I}$ e $\mathrm{D}+\mathrm{V}+\mathrm{I}$, foram administradas doses de insulina NPH U-100 para cada $100 \mathrm{~g}$ de rato diariamente. A dose de insulina administrada durante o tratamento está apresentada na Tabela 1. Para os ratos $\mathrm{D}+\mathrm{V}$ e $\mathrm{D}+\mathrm{V}+\mathrm{I}$, a água rica em vanádio foi colocada em garrafas de cor âmbar, para evitar sua degradação pela luz e seu consumo monitorado diariamente.

\section{Análise estatística}

Os dados foram submetidos à análise de variância de uma via-ANOVA com pós-teste de Tukey para um nível de significância de $p<0,05$.

\section{Resultados}

Inicialmente, foi administrado $1 \mathrm{U}$ de insulina $\mathrm{NPH} /$ rato diariamente. Devido à não estabilização da glicemia, foi necessário aumentar a dose (Tabela 1). A normalização da concentração glicêmica para o grupo D + I foi alcançada quando a dose de insulina foi elevada para $4 \mathrm{U} /$ rato, onde a glicemia atingiu $55 \mathrm{mg} / \mathrm{dL}$ (Tabela 2). O vanádio não mostrou eficácia em reduzir a concentração de glicose sangüínea dos grupos tratados (Tabela $2 \mathrm{e}$ Figura 1).

O consumo médio semanal de água pelos ratos está apresentado na Figura 2. O grupo controle ingeriu, em média, $50 \mathrm{~mL} /$ dia. $\mathrm{O}$ grupo diabético consumiu 2,5 vezes mais quando comparado ao controle. O tratamento com insulina dos animais diabéticos promoveu redução da ingestão, mas esta permaneceu 1,2 vezes maior que à do grupo controle $(\mathrm{p}<0,001)$ e $60 \%$ menor comparada à do não tratado $(\mathrm{p}<0,05)$. O grupo tratado com vanádio reduziu o consumo de água, permanecendo 1,7 vezes maior em relação ao do grupo controle $(\mathrm{p}<0,001)$. A combinação dos tratamentos de vanádio e insulina reduziu significativamente a ingestão hídrica em relação à do grupo não tratado $(\mathrm{p}<0,05)$.

A média do consumo de ração do grupo controle foi $23 \pm 1 \mathrm{~g} /$ dia (Figura 3). A diabetes incrementou este consumo quando comparado ao do grupo $\mathrm{C}(\mathrm{p}<0,001)$. $\mathrm{O}$ tratamento com insulina não alterou este quadro, permanecendo idêntico ao do grupo sem tratamento. $\mathrm{O}$ tratamento com vanádio não reduziu significativamente o consumo de ração. Comparando-se o grupo tratado com vanádio e insulina (D+V+I) com o grupo sem tratamento (D) e ao tratado somente com insulina $(\mathrm{D}+\mathrm{I})$, foi possível observar redução significativa na quantidade de alimento consumida $(\mathrm{p}<0,05)$.

A variação da massa corporal dos ratos, em porcentagem, ao longo do período experimental, está apresentada na Figura 4. O grupo controle incrementou sua massa corporal em $10 \%$. O grupo diabético perdeu $20 \%$, o qual foi mantido até o final do experimento. $\mathrm{O}$ tratamento com insulina impediu a perda de massa corporal durante as semanas de avaliação, quando comparada à do grupo sem tratamento $(\mathrm{p}<0,001)$. Os ratos tratados com vanádio apresentaram redução de $10 \%$ da massa corporal na primeira semana de tratamento, com incremento de $5 \%$ até o período final $(\mathrm{p}<0,05)$. A combinação dos tratamentos de vanádio e insulina impediu a perda e incrementou em $15 \%$ da variação da massa corporal $(\mathrm{p}<0,05)$ quando comparada à do grupo tratado somente com vanádio $(\mathrm{D}+\mathrm{V})$. 
Tabela 1 - Doses de insulina administradas durante os 16 dias de tratamento

\begin{tabular}{|c|c|c|c|c|c|c|c|c|c|c|c|c|c|c|c|c|}
\hline Dose & & & & & & & & & ias & & & & & & & \\
\hline & 1 & 2 & 3 & 4 & 5 & 6 & 7 & 8 & 9 & 10 & 11 & 12 & 13 & 14 & 15 & 16 \\
\hline $1 U$ & $X$ & $X$ & & & & & & & & & & & & & & \\
\hline $2 U$ & & & $X$ & $X$ & $X$ & $X$ & $X$ & & & & & & & & & \\
\hline $3 U$ & & & & & & & & $x$ & $x$ & $x$ & $x$ & $X$ & $x$ & $x$ & & \\
\hline $4 U$ & & & & & & & & & & & & & & & $X$ & $X$ \\
\hline
\end{tabular}

Tabela 2 - Glicemia dos animais diabéticos tratados ou não com água rica em vanádio pelo período de 16 dias, onde C representa animais controle, $D$ diabéticos não tratados, $D+I$ diabéticos tratados com insulina, $D+V$ diabéticos tratados com vanádio $e$ $D+V+I$ diabéticos tratados com insulina e vanádio. Os dados estão expressos em mg/dL e representam a média \pm erro padrão da média de 5 animais por grupo. ${ }^{a} p<0,001$ vs. $C ;{ }^{b} p<0,05$ vs. $D+I$

\begin{tabular}{ccccccc}
\hline \multicolumn{7}{c}{ Dias } \\
\hline Grupos & 0 & 2 & 4 & 7 & 14 & 16 \\
\hline C & $144 \pm 4,65$ & $104 \pm 13,46$ & $103 \pm 9,62$ & $124 \pm 9,62$ & $127 \pm 7,78$ & $98 \pm 11,07$ \\
D & $576 \pm 19,69$ & $593 \pm 67,46$ & $559 \pm 19,53$ & $640 \pm 25,60$ & $527 \pm 12,38$ & $584 \pm 23,88$ \\
& $a$ & $a$ & $a$ & $a$ & $a$ & $a b$ \\
D + I & $471 \pm 23,53$ & $558 \pm 58,16$ & $426 \pm 52,98$ & $392 \pm 68,95$ & $348 \pm 86,37$ & $55 \pm 2,64^{a}$ \\
& $a$ & $a$ & $a$ & $a$ & $a$ & \\
$D+V$ & $509 \pm 22,81$ & $562 \pm 17,08$ & $480 \pm 25,68$ & $561 \pm 20,90$ & $582 \pm 30,87$ & $584 \pm 74,26$ \\
& $a$ & $a$ & $a$ & $a$ & $a$ & $a b$ \\
D + V + I & $495 \pm 5,06$ & $514 \pm 21,28$ & $366 \pm 58,67$ & $495 \pm 44,89$ & $399 \pm 55,05$ & $211 \pm 76,15$ \\
& & $a$ & $a$ & $a$ & $a$ & $a b$
\end{tabular}




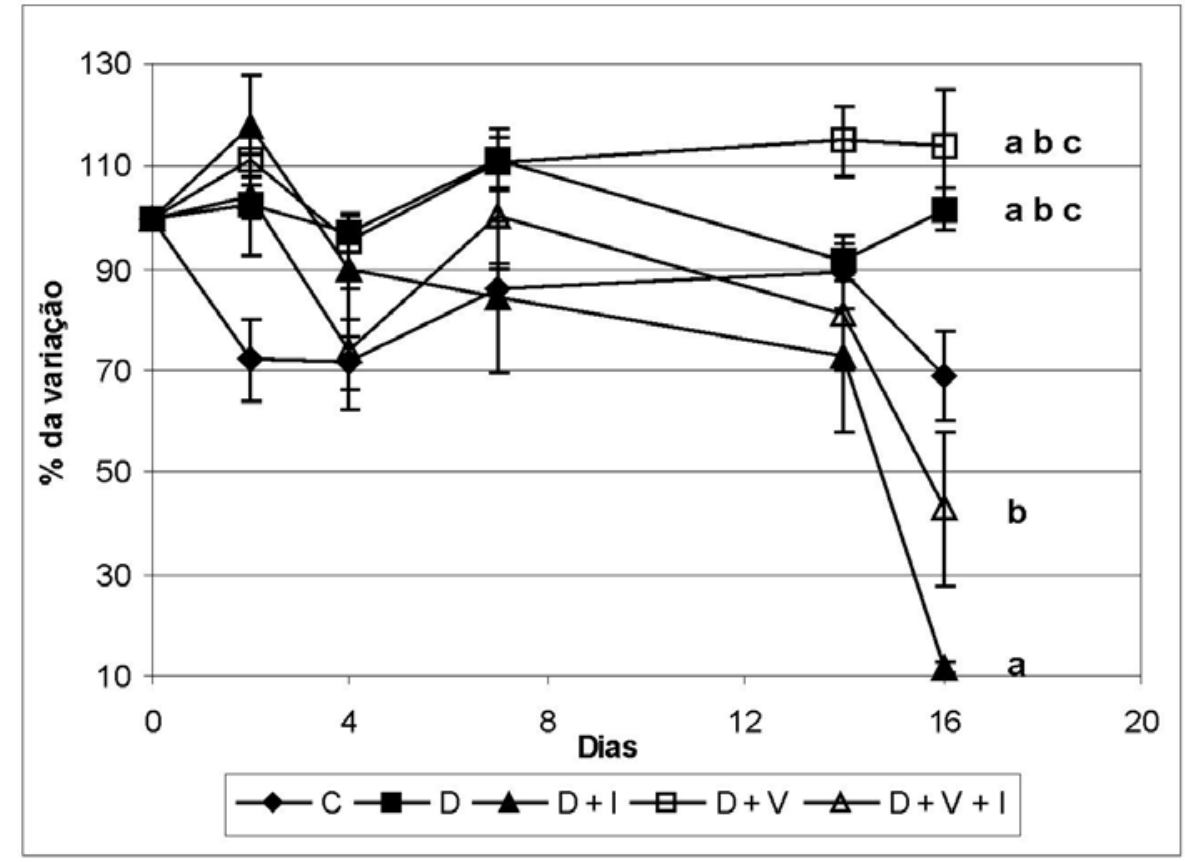

Figura 1 - Porcentagem da variação da glicemia de ratos controle (C), diabético (D), diabético tratado com insulina $(D+I)$, diabético tratado com vanádio $(D+V)$ e diabético tratado com vanádio e insulina $(D+V+I)$. Dados expressos como média \pm erro padrão da média. ${ }^{a} p<$ 0,05 vs. $C$; ${ }^{b} p<0,001$ vs. $D+I e^{c} p<0,05$ vs. $D+V+I$

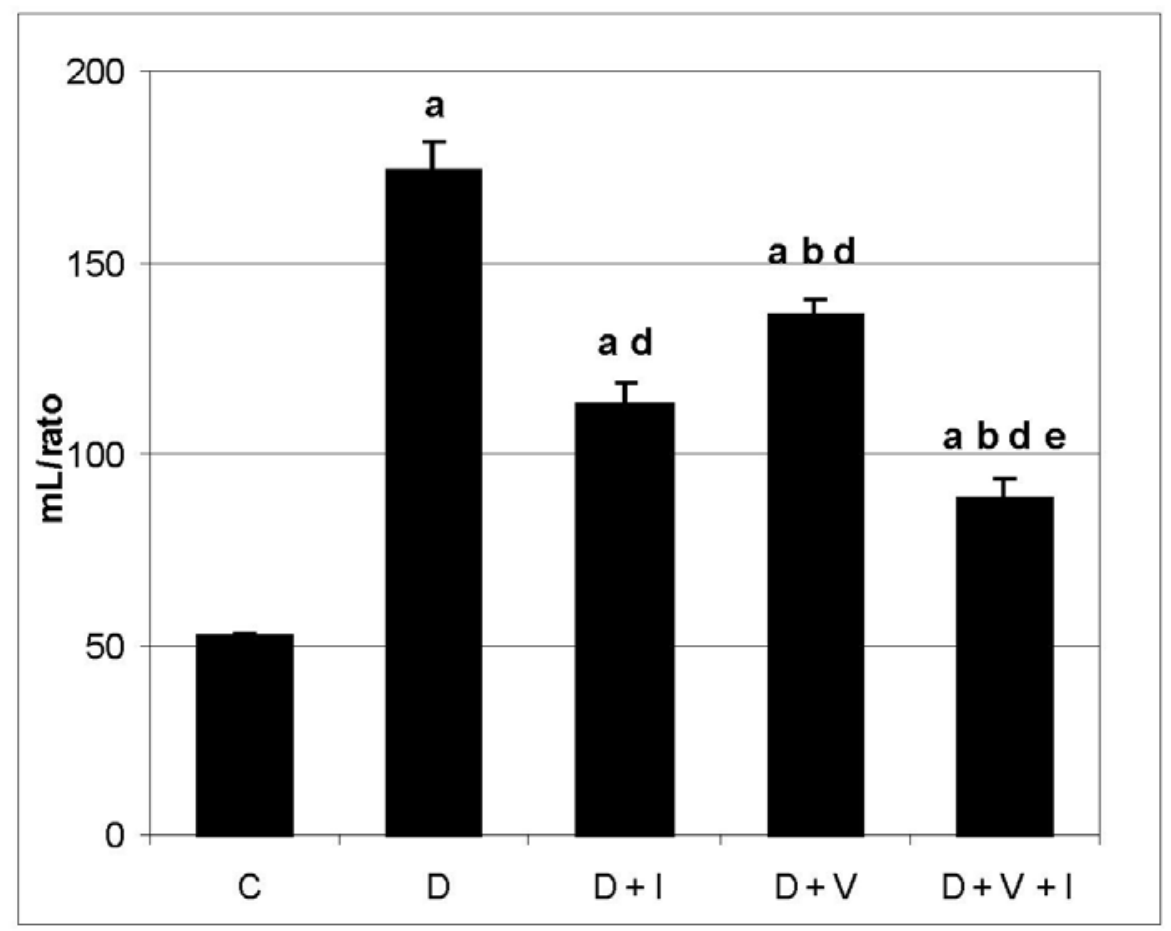

Figura 2 - Média do consumo de água (mL/rato) ao longo dos 16 dias dos animais dos grupos controle $(C)$, diabético $(D)$, diabético tratado com insulina $(D+I)$, diabético tratado com vanádio $(D+V)$ e diabético tratado com vanádio e insulina $(D+V+I)$. Dados expressos como média \pm erro padrão da média. ${ }^{a} p<0,001$ vs. $C ;{ }^{b} p<0,001$ vs. $D+I ;{ }^{d} p<0,05$ vs. $D e^{e} p<0,001$ vs. $D+V$ 


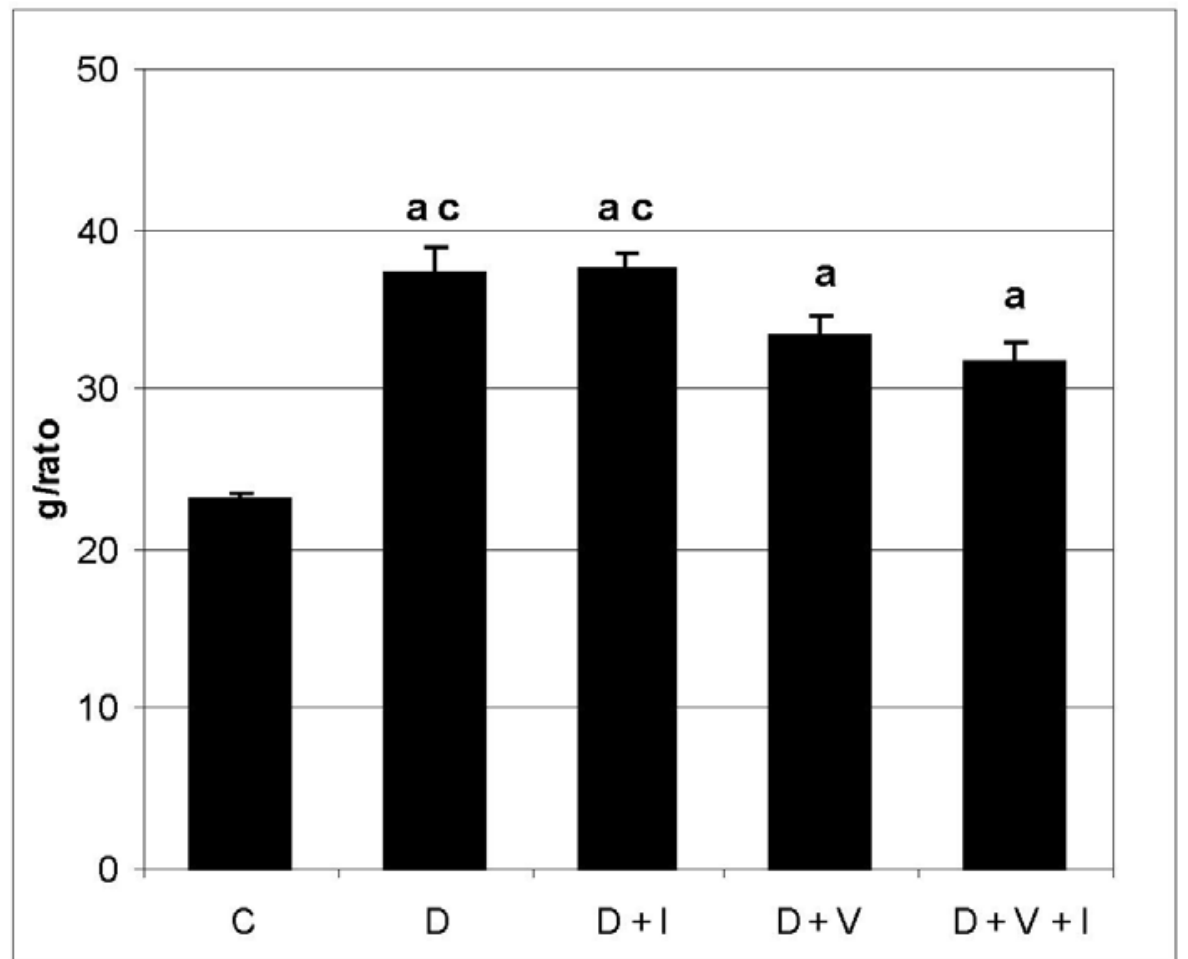

Figura 3 - Média do consumo de reação (g/rato) aolongo dos 16 dias dos animais dos grupos controle $(C)$, diabético $(D)$, diabético tratado com insulina $(D+I)$, diabético tratado com vanádio $(D+V)$ e diabético tratado com vanádio e insulina $(D+V+I)$. Dados expressos como média \pm erro padrão da média. ${ }^{a} p<0,001$ vs. $C e^{c} p<0,05$ vs. $D+V+I$

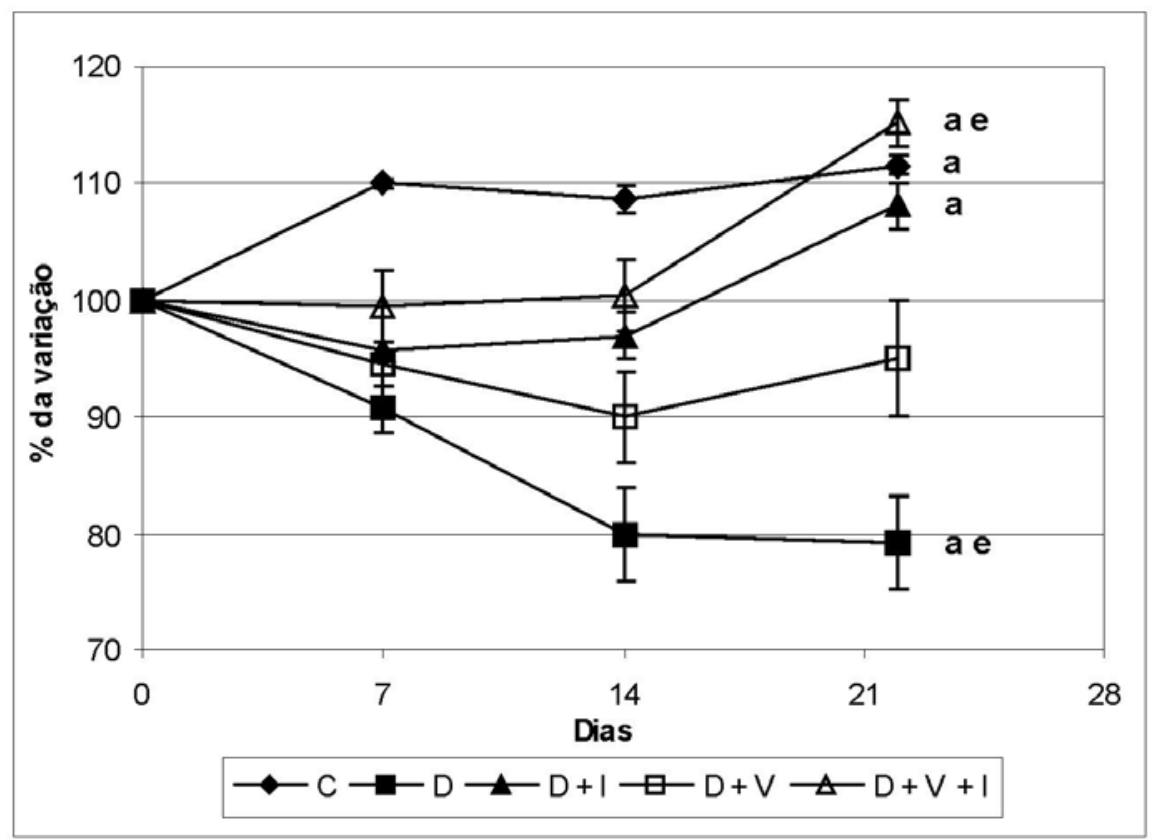

Figura 4- Porcentagem da variação do peso, semanal, de ratos controle (C), diabético (D), diabético tratado com insulina $(D+I)$, diabético tratado com vanádio $(D+V)$ e diabético tratado com vanádio e insulina $(D+V+I)$. Dados expressos como média \pm erro padrão da média. ${ }^{a} p<0,001$ vs. $C e^{e} p<0,05$ vs. $D+V$ 


\section{Discussão}

Existe grande número de modelos animais disponíveis para experimentação dos efeitos das drogas indutoras de diabetes. Injeções de aloxana e estreptozotocina induzem quimicamente ao modelo de diabetes mellitus tipo I, caracterizado por hiperglicemia, hipoinsulinemia, hiperlipidemia, hiperfagia e polidipsia (11). Animais aqui estudados apresentavam hiperglicemia (Tabela 2 e Figura 1), polidipsia (Figura 2) e hiperfagia (Figura 3), confirmando estado diabético do tipo I. Nesse modelo, o vanádio tem sido efetivo em normalizar a concentração de glicose plasmática e restaurar as concentrações alteradas de lipídios plasmáticos (11).

O efeito "anti"-diabético dos sais de vanádio foi primeiramente relatado em 1899, 22 anos antes da descoberta da insulina (18). Quase 100 anos depois, o interesse no vanádio ressurgiu quando Heyliger et al. (19) demonstraram in vivo o efeito redutor de glicemia dos compostos de vanádio administrados oralmente em ratos diabéticos por estreptozotocina.

Vários estudos subseqüentes demonstraram que o vanádio mimetiza a ação da insulina, in vitro e in vivo, alterando o metabolismo de carboidratos, proteínas e lipídios (revisado em 20). Alguns efeitos, in vitro, do vanádio incluem a estimulação do transporte de glicose $(21,22,23)$, glicólise e oxidação da glicose $(24,25)$, síntese de glicogênio (23), lipogênese, inibição da lipólise (26) e gliconeogênese (27). Os experimentos, in vivo, foram estudados extensivamente em diferentes modelos animais com diabetes. Ratos diabéticos por estreptozotocina tratados com vanádio demonstraram baixas concentrações de glicose, normalização da saída de glicose hepática, correção das enzimas glicolíticas, restauração da síntese de glicogênio e da atividade fosforilase, normalização da expressão do transportador de glicose e melhora do estresse oxidativo (28). Estas propriedades fazem do vanádio uma nova ferramenta para o tratamento em potencial para diabetes tipo II e a síndrome metabólica $(29,30,31)$. Em estudo quase recente (32), ao administrarem peróxido de vanadil e peróxido de vanadato a ratos diabetizados, encontraram redução significativa da polidipsia, bem como da percentagem da variação da glicemia. Estes dados foram corroborados por nossos dados aqui.

Para Verma et al. (28), a capacidade do vanádio em causar hipoglicemia sem aumentar as concentrações plasmáticas de insulina na diabetes tipo II é indicativo de sua habilidade em aumentar a sensibilidade à insulina. Muitos estudos mostraram que a concentração plasmática de glicose em ratos diabéticos tratados com vanádio foi restaurada aos valores normais, sem elevação das concentrações circulantes de insulina. O tratamento também reduziu as concentrações plasmáticas de insulina em animais controle tratados, sem alteração dos valores de glicose plasmática. Em adição, o vanádio foi responsável por melhorar o controle glicêmico em humanos com diabetes mellitus $(11,32,33)$.

Os compostos de vanádio podem ser aplicados na terapia oral da diabetes como substitutos ou em combinação com a insulinoterapia para reduzir a dose de insulina requerida (28). Estudos sugerem que além de diminuir a necessidade de insulina, o vanádio pode melhorar o estado metabólico em diabetes tipo I. De fato, em muitos casos, não é necessária a reposição de insulina $(13,28,34)$.

A dose de insulina foi ajustada durante o período experimental (Tabela 1). Somente quando utilizamos $4 \mathrm{U} /$ rato observamos redução significativa da glicemia para animais do grupo D + I (Tabela 2). A baixa reação da insulina NPH pode estar associada ao seu pico de ação (6 horas). Como as mensurações da glicemia foram realizadas após este período, provavelmente o pico de ação havia passado. A opção por aplicar uma dose diária também pode ajudar a explicar a não verificação da queda da glicemia. Porém, o mesmo não foi observado para os ratos tratados com vanádio e insulina $(\mathrm{D}+\mathrm{V}+\mathrm{I})$ (Tabela 2 e Figura 1). Sabe-se que o vanádio aumenta a sensibilidade. Assim, é possível que a glicemia tenha sido reduzida bem antes do momento da mensuração e isso poderia explicar o valor de $211,3 \mathrm{mg} / \mathrm{dL}(\mathrm{D}+\mathrm{V}+\mathrm{I})$, que talvez não estivesse em queda e sim em elevação após a ação da insulina mais vanádio.

A concentração de vanádio necessária diariamente para redução significativa da glicemia não foi definida. Existem muitas variantes que interferem nessa determinação, seja a duração do tratamento até concentração e forma de vanádio que são utilizadas. Em vários estudos, sais de vanádio são administrados oralmente por meio da água e o efeito redutor da glicemia foi visto entre 2-4 dias depois do início do tratamento. A dose de sais de vanádio requerida para efeito máximo da ação redutora variou nestes estudos, mas a concentração média de $0,5 \mathrm{mg} / \mathrm{mL}$ na água mostrou ser suficiente $(15,33)$.

Em estudo realizado por Meyerovitch et al. (35), a alta concentração de vanadato administrada oralmente $(0,8 \mathrm{mg} / \mathrm{mL}$ na água) reduziu as 
concentrações de glicose sangüínea, resultando no aparecimento de hipoglicemia nos animais teste entre 2-4 dias. Uma menor concentração de vanadato $(0,2$ $\mathrm{mg} / \mathrm{mL}$ na água) também reduziu as concentrações de glicose sangüínea em 4 dias, mas não levou a hipoglicemia em pelo menos 3 semanas. Os efeitos do vanadato foram reversíveis e a hiperglicemia retornou dois dias depois da retirada do vanadato (35). No estudo de Brichard e Henquin (5), a baixa concentração de vanadato $(0,08 \mathrm{mg} / \mathrm{mL})$ não foi eficaz em reduzir glicose sangüínea. Este dado se aproximou muito dos resultados obtidos neste trabalho. A concentração de vanádio presente na água (próxima a $0,04 \mathrm{mg} / \mathrm{L}$ de sais de vanádio) proveniente de Ibirá pode ter sido motivo da não redução da glicemia. Mesmo sendo rica em sais de vanádio, esta água, quando comparada a outras, tem concentração baixa se comparada com dados da literatura.

Experimento realizado por Badmaev et al. (12) revelou que o tratamento com complexo de vanádio orgânico (dose de $30 \mathrm{mg} / 100 \mathrm{~mL}$ ) administrado por 15 dias aos ratos diabéticos reduziu a ingestão hídrica, concentração de glicose sangüínea, colesterol e triglicérides. Os dados aqui obtidos mostraram que o tratamento com a água rica em vanádio (concentração de $0,042 \mathrm{mg} / \mathrm{L}$ ) reduziu significativamente a ingestão hídrica após administração por 16 dias (Figura 2).

Poucheret et al. (36) relataram que compostos de vanádio são capazes de normalizar a hiperfagia associada a diabetes experimental. McNeill et al. (11) demonstraram que tratamento com vanadato reduziu tanto a ingestão alimentar quanto a hídrica em ratos diabéticos, porém não afetou o ganho de massa corporal destes animais. Resultados aqui obtidos revelaram que o tratamento com água rica em sais de vanádio não reduziu significativamente o consumo de ração (Figura 3), mas reduziu a perda de massa corporal quando comparada à do grupo diabético (Figura 4).

Os efeitos tóxicos mais comuns do vanádio incluem diarréia, redução da ingestão hídrica e alimentar, desidratação e redução do ganho de massa corporal $(15,33)$. Não foram observados sinais da toxicidade do vanádio, provavelmente pela baixa concentração presente na água de Ibirá e por estar mais relacionada aos compostos inorgânicos de vanádio. Um importante avanço para os tratamentos já existentes seria determinar uma dosagem terapêutica eficaz, o que evitaria a toxicidade significativa associada aos compostos de vanádio.
Os dados obtidos neste trabalho sugerem que somente a ingestão de água rica em vanádio como terapia não é suficiente para o tratamento da diabetes mellitus. Apesar de melhora indicativa por meio de alguns sintomas, a concentração de glicose plasmática não foi normalizada. Assim, mais estudos com fontes naturais, que exibam maior concentração de vanádio, podem ser importantes para se definir o papel da ingestão da água destas fontes como coadjuvantes na terapia da diabetes.

\section{Referências}

1. Emilien G, Maloteaux JM, Ponchon M. Pharmacological management of diabetes: recent progress and future perspective in daily drug treatment. Pharmacology \& Therapeutics. 1999; 81(1):37-51.

2. Haber EP, Curi R, Carvalho CRO, Carpinelli AR. Secreção da insulina: efeito autócrino da insulina e modulação por ácidos graxos. Arquivos Brasileiros de Endocrinologia e Metabologia. 2001; 45(3):219-227.

3. Thompson KH. Vanadium and diabetes. Biofactors. 1999; 10:43-51.

4. Bell GI, Polonsky KS. Diabetes mellitus and genetically programmed defects in beta-cell function. Nature. 2001; 414:788-791.

5. Brichard SM, Henquin JC. The role of vanadium in the management of diabetes. Trends in Pharmacological Sciences. 1995; 16:265-270.

6. Brownlee M. Biochemistry and molecular cell biology of diabetic complications. Nature. 2001; 414:813-820.

7. American Diabetes Association. Diagnosis and classification of Diabetes Mellitus. Diabetes Care. 2005; 28:37-42.

8. Gross JL, Silveiro SP, Camargo JL, Reichelt AJ, Azevedo MJ. Diabetes Melito: diagnóstico, classificação e avaliação do controle glicêmico. Arquivos Brasileiros de Endocrinologia e Metabologia. 2002; 46(1):16-26.

9. Mouser J. New drugs for management of diabetes: insulin analogues, inhaled insulin, pramlintide, and novel peptides. Nutritional in Clinical Practice. 2004; 19:172-180. 
10. Stolar MW. Insulin resistance, diabetes and the adipocyte. American Journal of Health-System Pharmacy. 2002; 59:S3-S8.

11. McNeill JH, Yuen, VG, Dai, S, Orvig C. Increased potency of vanadium using organic ligands. Molecular and Cellular Biochemistry. 1995; 153:175-180.

12. Badmaev V, Prakash S, Majeed M. Vanadium: a review of its potential role in the fight against diabetes. The Journal of Alternative and Complementary Medicine. 1999; 5(3):273-291.

13. Marzban L, McNeill JH. Insulin-like actions of vanadium: potential as a therapeutic agent. The Jounal of Trace Elements in Experimental Medicine. 2003; 16:253-267.

14. Preet A, Gupta BL, Yadava PK, Baquer NZ. Efficacy of lower doses of vanadium in restoring altered glicose metabolism and antioxidant status in diabetic rat lenses. Journal of Biosciences. 2005; 30(2):221-230.

15. Srivastava AK. Anti-diabetic and toxic effects of vanadium compounds. Molecular and Cellular Biochemistry. 2000; 206:177-182.

16. Sacks DB, Bruns DE, Goldstein DE, Maclaren NK, McDonald JM, Parrot M. Guidelines and recommendations for laboratory analysis in the diagnosis and management of diabetes mellitus. Clinical Chemistry. 2002; 48:436-472.

17. Trinder R. Determination of glucose in blood using glicose with alternative oxygen acceptor. Annals of Clinical Biochemistry. 1969; 6:24-27.

18. Lyonnet B, Martz M. L'emploi thérapeutique des dérivés du vanadium. La Presse Medicale. 1899; 32:191-192.

19. Heyliger C, Tahiliani A, McNeill J. Effect of vanadate on elevated blood glucose and depressed cardiac performance of diabetic rats. Science. 1985; 22(4693):1474-1477.

20. Cam M, Brownsey R, Mcneill J. Mechanisms of vanadium action: Insulin-mimetic or insulinenhancing agent? Canadian Journal of Physiology and Pharmacology. 2000; 78:829-847.

21. Carey J, Azevedo J, Morris P, Pories W, Dohm G. Okadaic acid, vanadate, and phenylarsine oxide stimulate 2-deoxyglucose transport in insulin-resistant human skeletal muscle. Diabetes. 1995; 44:682-688.
22. Dubyak G, Kleinzeller A. The insulin-mimetic effects of vanadate in isolated rat adipocytes. Dissociation from effects of vanadate as a $\mathrm{Na} / \mathrm{K}$ ATPase inhibitor. Journal of Biological Chemistry. $1980 ; 255: 5306-5312$.

23. Clark A, Fagan J, Mitch W. Selectivity of the insulin-like actions of vanadate on glucose and protein metabolism in skeletal muscle. Biochemical Journal. 1985; 232:273-276.

24. Shechter Y, Karlish S. Insulin-like stimulation of glucose oxidation in rat adipocytes by vanadyl (IV) ions. Nature. 1980; 284:556-558.

25. Duckworth W, Solomon S, Liepnieks J, Hamel F, Hand S, Peavy D. Insulin-like effects of vanadate in isolated rat adipocytes. Endocrinology. 1988; 122:2285-2289.

26. Degani I, Gochin M, Karlish S, Shechter Y. Electron paramagnetic resonance studies and insulin-like effects of vanadium in rat adipocytes. Biochemistry. 1981; 20(20): 5795-5799.

27. Bosch F, Iatzoglov M, Park E, Hanson R. Vanadate inhibits expression of the gene for phosphoenolpyruvate carboxykinase (GTP) in rat hepatoma cells. The Journal of Biological Chemistry. 1990; 265:13677-13682.

28. Verma S, Cam MC, McNeill JH. Nutritional factors that can favorably influence the glicose/ insulin system: vanadium. Journal of the American College of Nutrition. 1998; 17(1):11-18.

29. Bailey C. Potencial new treatments for type 2 diabetes. Trends in Pharmacological Sciences. 2000; 21:259-265.

30. Moller D. New drug targets for type 2 diabetes and the metabolic syndrome. Nature. 2001; 414:821-827.

31. Balasubramanyam M, Mohan V. Orally active insulin mimics: where do we stand now? Journal of Biosciences. 2001; 26:383-390.

32. Yamazaki RK, Hirabara SM, Tchaikovski Jr O, Lopes MCP, Nogata C, Aikawa J, et al. The effects of peroxovanadate and peroxovanadyl on glucose metabolism in vivo and identification of signal transduction proteins involved in the mechanism of action in isolated soleus muscle. Mollecular and Cellular Biochemistry. 2005; 273:145-150. 
33. Fantus G, Tsiani E. Multifuncional actions of vanadium compounds on insulin singnaling pathways: Evidence for preferential enhacement of metabolic versus mitogenic effects. Molecular and Cellular Biochemistry. 1998; 182:109-119.

34. Srivastava AK, Mehdi MZ. Insulino-mimetic and anti-diabetic effects of vanadium compounds. Diabetic Medicine. 2005; 22(1):2-13.

35. SchechterY, Goldwaser I, Mironchik M, Fridkin M, Gefel D. Historic perspective and recent developments on the insulin-like action of vanadium; toward developing vanadium-based drugs for diabetes. Coordination Chemistry Reviews. 2003; 237:3-11.
36. Meyerovitch J, Farfel Z, Sack J, Shechter Y. Oral administration of vanadate normalizes blood glicose levels in streptozotocin-treated rats. The Journal of Biological Chemistry. 1987; 262(14):6658-6662.

37. Poucheret P, Verma S, Grynpas MD, McNeill JH. Vanadium and diabetes. Molecular and Cellular Biochemistry. 1998; 188:73-80.

Recebido em/Received in: August 01, 2006

Aceito em/Accepted in: September 05, 2006 